Schmerz 2006 $20: 481-489$

DOI 10.1007/s00482-006-0472-9

Online publiziert: 22. Februar 2006

(c) electronic version (html)

Springer Medizin Verlag 2006

(c) print \& online pdf

Deutsche Gesellschaft zum Studium des

Schmerzes 2006. Published by Springer

Medizin Verlag - all rights reserved

J. C. Türp ${ }^{1}$ A. Hugger ${ }^{2}$. P. Nilges ${ }^{3}$. S. Hugger ${ }^{4}$ J. Siegert ${ }^{3} \cdot$ E. Busche $^{5}$.

S. Effenberger ${ }^{6} \cdot$ H. J. Schindler ${ }^{7}$

${ }^{1}$ Klinik für Rekonstruktive Zahnmedizin und Myoarthropathien,

Universitätskliniken für Zahnmedizin, Basel

${ }^{2}$ Poliklinik für Zahnärztliche Prothetik, Westdeutsche

Kieferklinik, Universität Düsseldorf

${ }^{3}$ DRK-Schmerzzentrum Mainz

${ }^{4}$ Poliklinik für Zahnärztliche Prothetik, Zentrum für Zahn-

, Mund- und Kieferheilkunde, Universität Münster

${ }^{5}$ Fakultät für Zahn-, Mund- und Kieferheilkunde, Universität Witten/Herdecke

${ }^{6}$ Zentrum für Zahnmedizin, Campus-Virchow-Klinikum, Universitätsmedizin Berlin

${ }^{7}$ Bundesforschungsanstalt für Ernährung, Karlsruhe

\title{
Aktualisierung der Empfehlungen zur standardisierten Diagnostik und Klassifikation von Kaumuskel- und Kiefergelenkschmerzen
}

wohl somatische (Achse I) als auch psychosoziale Aspekte (Achse II) diagnostiziert.

\section{Mindestdiagnostik}

Bei Verdacht auf eine schmerzhafte Myoarthropathie sollte zunächst eine Mindestdiagnostik erfolgen (1. Stufe). Sie beinhaltet obligat:

- eine gezielte, schmerzbezogene Anamnese (u. a. Schmerzbeginn, Schmerzlokalisation anhand von Ganzkörperschemata, Schmerzdauer, Schmerzhäufigkeit, Schmerzbeeinträchtigung);

- die physische Befundung des Kausystems, bestehend aus Messung der Unterkieferbeweglichkeit (v. a. maximale Kieferöffnung als Summe des vertikalen Überbisses und der maximal möglichen Schneidekantendistanz), Bestimmung der Palpationsempfindlichkeit von Kaumuskeln und Kiefergelenken (Achse I) und Überprüfung der Okklusion;

Die Empfehlungen [83] fußen auf dem Prinzip einer Stufendiagnostik; es werden eine Mindest-, eine Standard- sowie eine erweiterte Diagnostik unterschieden (- Abb. 1). Auf jeder Stufe werden so-

\section{Zusammenfassung der Empfehlungen}

- ein schmerzbezogenes psychosoziales Screening anhand der Graduierung chronischer Schmerzen (GCS), der deutschen Version des Graded Chro- nic Pain Status nach Von Korff et al.

([97]; Achse II);

- eine Panoramaschichtaufnahme.

\section{Standarddiagnostik}

Die Standarddiagnostik (2. Stufe) besteht aus den Einzelschritten der 1. Stufe, verbunden mit einer erweiterten psychosozialen Diagnostik zur Beurteilung des Ausmaßes einer evtl. vorhandenen depressiven Verstimmung sowie unspezifischer somatischer Symptome als Hinweis auf globale Beeinträchtigung des subjektiven Befindens (Achse II). In spezialisierten Behandlungseinrichtungen (Praxen oder Kliniken) sollte diese differenzierte Diagnostik auf jeden Fall erfolgen.

Sie ist weiterhin zu empfehlen, wenn

- eine starke schmerzbezogene Beeinträchtigung vorhanden ist (GCSGrad III oder IV: dysfunktionaler Schmerz) oder

- trotz unauffälligem GCS (Grad o, 1 oder 2: kein oder funktionaler Schmerz) anamnestische Hinweise

Die Autoren schreiben im Namen des Interdisziplinären Arbeitskreises für Mund- und Gesichtsschmerzen der Deutschen Gesellschaft zum Studium des Schmerzes 


\section{Übersichten}

\begin{tabular}{|c|c|c|c|}
\hline Deutschsprachiges Schrifttum & Englischsprachiges Schrifttum & $\begin{array}{l}\text { International Association for the } \\
\text { Study of Pain (IASP) }\end{array}$ & $\begin{array}{l}\text { Medline (Medical Subject Hea- } \\
\text { dings) }\end{array}$ \\
\hline $\begin{array}{l}\text { Funktionsstörungen des Kauorgans } \\
\text { [39] }\end{array}$ & $\begin{array}{l}\text { Temporomandibular disorders } \\
\text { (TMD, TMDs; }[11,60] \text { ) }\end{array}$ & $\begin{array}{l}\text { Temporomandibular pain and } \\
\text { dysfunction syndrome (Code } 034 . \\
\text { X8a; [55]) }\end{array}$ & $\begin{array}{l}\text { Temporomandibular joint } \\
\text { dysfunction syndrome }\end{array}$ \\
\hline $\begin{array}{l}\text { Kraniomandibuläre Dysfunktionen } \\
\text { (CMD; v. a. in Deutschland) }\end{array}$ & $\begin{array}{l}\text { Craniomandibular disorders (CMD, } \\
\text { CMDs; [54]) }\end{array}$ & - & Craniomandibular dsorders \\
\hline $\begin{array}{l}\text { Myoarthropathien des Kausystems } \\
{[63,72] \text {; in der Schweiz, z. T auch in }} \\
\text { Deutschland }\end{array}$ & Facial arthromyalgia [17] & - & Temporomandibular joint disorders \\
\hline Costen-Syndrom (obsolet) & - & - & - \\
\hline
\end{tabular}

auf psychosoziale Belastung vorliegen oder

- Schmerzen zum Zeitpunkt der Patientenvorstellung länger als 6 Monate (häufig als Zeitschwelle für Chronifizierung definiert) bestehen oder

- es nach einer alio loco oder selbst durchgeführten Initialtherapie von 4 Wochen nicht zu einer wesentlichen Besserung gekommen ist.

\section{Erweiterte Diagnostik}

Bei besonderen Fragestellungen (z. B. Abklärung möglicher Risikofaktoren) können im Rahmen der erweiterten Diagnostik (3. Stufe) weitere diagnostische Methoden und Verfahren zur Anwendung kommen. Dazu zählen u. a. spezielle bildgebende Verfahren wie konventionelle Tomographie, Computertomographie und Magnetresonanztomographie, Untersuchungstechniken wie die manuelle und die instrumentelle Funktionsanalyse, die klinische und die instrumentelle Okklusionsanalyse (alle Achse I) sowie die Erfassung schmerzassoziierter Parameter wie Lebensqualität, Schmerzverhalten, kognitive Schmerzverarbeitung und Schmerzbewältigung (Achse II).

\section{Bestandsaufnahme}

Die vom Arbeitskreis ausgesprochenen Empfehlungen, die in der Zwischenzeit auch in zahnärztlichen Fachzeitschriften vorgestellt wurden $[82,85]$, erfuhren in der Fachliteratur ein positives Echo (z. B. $[48,65])$.

Unsere im Jahr 2000 veröffentlichte Publikation [83] war die erste, die das Diagnostik- und Klassifikationssche- ma Research Diagnostic Criteria for Temporomandibular Disorders (RDC/TMD; [11]) im deutschsprachigen Raum vorstellte und - in einer leicht modifizierten Form - in die Empfehlungen integrierte. Die international in hohem Maße anerkannten RDC/TMD (http://www.rdctmd-consortium.org) werden auch hierzulande immer mehr gewürdigt $[47,48$, 52, 71, 78]. Ihr großes Plus besteht darin, dass sie das diagnostische Vorgehen am Patienten standardisieren und $u$. a. detaillierte Hinweise für das Vorgehen bei der klinischen Befundung geben. Dass für die alltägliche Praxis dringend Standards erforderlich sind, mit denen eine qualitativ ausreichende schmerztherapeutische Versorgung nach aktuellem Kenntnisstand ermöglicht wird, belegten unter anderem die Ergebnisse der Untersuchung von Wirz et al. [99].

Ebenfalls neu war die Einführung eines diagnostischen Stufenschemas (Unterteilung in Mindest-, Standard- und erweitere Diagnostik). Andere deutsche zahnmedizinische Arbeitsgruppen haben dieses Prinzip inzwischen ebenfalls eingeführt und für ihre Bedürfnisse adaptiert [18].

\section{Aktualisierungen}

\section{Klassifikation}

Aktuelle Studien belegen, dass die Prävalenz des Kaumuskelschmerzes in Patientenpopulationen 2- bis 3-mal höher ist als die des Kiefergelenkschmerzes [96, 100]. In zufälligen Bevölkerungsstichproben kann dieses Verhältnis noch deutlich ausgeprägter sein (z. B. in einer finnischen Studie [67]: Kaumuskelschmerz 13,3\%, Kiefergelenkschmerz 1,6\%).

\section{Mindestdiagnostik}

\section{Schmerzanamnese}

Eine umfassende Schmerzanamnese gilt zusammen mit der klinischen Untersuchung (vgl. [79]) als eines der verlässlichsten Instrumente der Erstbefundung [26]. Für die Schmerzdiagnostik ist sie die entscheidende Grundlage [57, 59, $74,101]$. Im Idealfall dienen die über die Schmerzanamnese hinausgehenden Untersuchungen (• Tab. 2) nur noch der Diagnosebestätigung [26].

Aufgrund der immensen Bedeutung der Schmerzanamnese sollte die Schulung in ärztlicher Gesprächsführung stärker gefördert werden. Schon einfache Veränderungen in der Gesprächsführung (z. B. das Stellen offener Fragen; das Zulassen auch von bizarr anmutenden Schilderungen und Kausalvorstellungen sowie von Gesprächspausen) führen zu messbaren Verbesserungen der Patientenbeteiligung am Anamnesegespräch [45].

$\mathrm{Zu}$ erfragen sind auch psychosoziale Belastungsfaktoren vor und zum Zeitpunkt des Schmerzbeginns. Um das Ausmaß der seelischen Belastung einzuschätzen, ist oft der Weg über den ,imaginären Dritten“ geeignet, dem Patienten diesen (zumindest beim Zahnarzt) unerwarteten Gesprächsverlauf zu erleichtern: „Viele Patienten, die solche Beschwerden haben, berichten, dass... Ist das bei Ihnen auch so?“

\section{Schmerzfragebogen}

In Anlehnung an den Deutschen Schmerzfragebogen der Deutschen Gesellschaft zum Studium des Schmerzes (DGSS) wurde speziell für die Diagnostik von $\mathrm{Pa}$ tienten mit persistierenden orofazialen Schmerzen ein 14-seitiger strukturierter 
Hier steht eine Anzeige.

算 Springer 

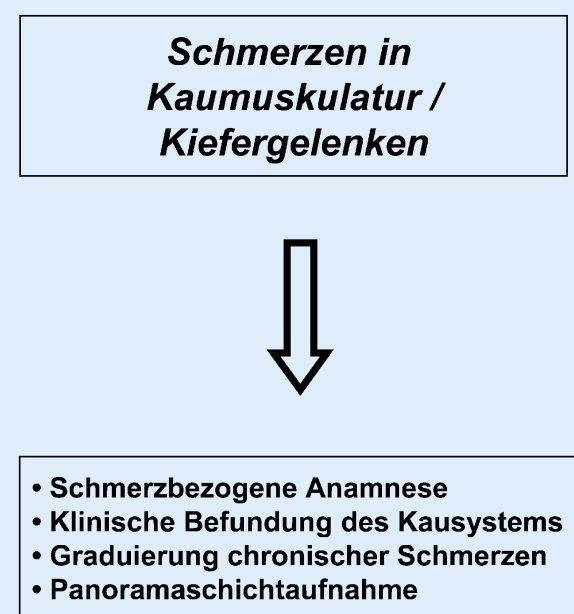

Mindestdiagnostik

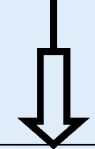

- Starke schmerzbezogene Beeinträchtigung (dysfunktionaler Schmerz)

- Anamnestische Hinweise auf psychosoziale Belastung

- Schmerzen > 6 Monate

- Keine Besserung nach 4-wöchiger Behandlung alio loco

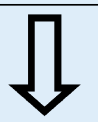

Zusätzlich:

Bestimmung des Ausmaßes von

- depressiver Verstimmung

- unspezifischen Beschwerden
Bei besonderen Fragestellungen

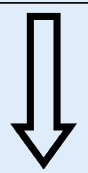

Zusätzlich je nach Bedarf:

- Erfassung weiterer

schmerzassoziierter Parameter

- Weitere bildgebende Verfahren

- Klinische Okklusionsanalyse

- Manuelle Funktionsanalyse

- Instrumentelle Funktionsanalyse

- Instrumentelle Okklusionsanalyse

Abb. 1 A Diagnostisches Stufenschema für Patienten mit Kaumuskel- und Kiefergelenkschmerzen

Schmerzfragebogen vorgestellt. Auf ihn und eine dazu gehörige Anleitung kann über das weltweite Netz kostenfrei zugegriffen werden [87]. Ziele dieses Instruments sind eine standardisierte Erfassung und Dokumentation gesichtsschmerzrelevanter Parameter sowie - im Sinne einer Filterdiagnostik - eine Abschätzung des Ausmaßes einer evtl. eingetretenen Schmerzchronifizierung.

Eine besondere Bedeutung kommt der Erfragung etwaiger Schmerzlokalisationen außerhalb des Gesichtsbereichs zu. Ein Patient, der einen Zahnarzt aufsucht, wird diese Schmerzregionen ungefragt meist nicht erwähnen [8o].

\section{Klinische Untersuchung}

Die Bestimmung der Unterkieferbeweglichkeit sowie der Palpationsempfindlichkeit der Kaumuskulatur (Mm. temporalis und masseter) und Kiefergelenke erfolgt nach einem standardisierten Protokoll gemäß der RDC/TMD [71]. Ein entsprechendes Befundblatt wurde vor kurzem vorgestellt [94]. Durch eine vorangehende Kalibrierung der Untersucher lässt sich die Reliabilität der Messungen deutlich erhöhen [37].
Kieferbeweglichkeit. Untersucher sollten damit rechnen, dass die maximale Kieferöffnung bei Männern größer ist als bei Frauen und mit zunehmendem Alter leicht abnimmt. In einer in Irland durchgeführten bevölkerungsrepräsentativen Studie ( $n=1513$, Alter 16-99 Jahre) betrug die durchschnittliche maximale Kieferöffnung bei Männern 43, bei Frauen $41 \mathrm{~mm}$. In der Altersgruppe der 16- bis 24-Jährigen lagen die entsprechenden Werte bei 44,6 bzw. 42,6 mm, in der Gruppe der über 64-Jährigen bei 41,o bzw. 39,o mm [19].

Die maximale Beweglichkeit des Unterkiefers nach rechts und links ist nur in seltenen Fällen gleich groß; die meisten Gesunden und Patienten (Rechtshänder) können den Unterkiefer weiter nach links als nach rechts schieben [91].

Dagegen sind bei einer vom Patienten berichteten schmerzlosen Einschränkung der Kieferbeweglichkeit, einer gemessenen maximalen Kieferöffnung von weniger als $35 \mathrm{~mm}$ und/oder einer über einen Zeitraum von wenigen Wochen oder Monaten erfolgten kontinuierlichen Verminderung der maximal möglichen Kieferöffnung unbedingt weitere diagnosti- sche Maßnahmen zu ergreifen (u. a. Ausschluss eines Tumors [93]).

Palpation. Vor der eigentlichen Untersuchung wird eine patientenindividuelle Kalibrierung durch Palpation der Mm. adductor pollicis und trapezius empfohlen; diese Betastung soll dem Patienten als Referenz bzgl. des zu erwartenden Drucks bei der anschließenden Palpation der Kaumuskulatur und Kiefergelenke dienen [27].

Unsere Entscheidung, die in der RDC/ TMD enthaltene Palpation des M. pterygoideus lateralis und der Regio postmandibularis (Mm. digastricus venter posterior und stylohyoideus) aufgrund fehlender diagnostischer Valididät nicht in die Empfehlungen aufzunehmen, erfuhr durch 2 neuere Publikationen Unterstützung. Demnach sind aus anatomischen Gründen weder der hintere Bauch des M. digastricus [93] noch - bis auf seltene Ausnahmen [76] - der untere Kopf des M. pterygoideus lateralis [86] palpierbar. Zudem ist die digitale Palpation dieser beiden Regionen auch bei beschwerdefreien Personen meist schmerzhaft; der diagnostische Wert einer solchen Palpa- 
tion wäre bereits aus diesem Grund fraglich.

Hinsichtlich des bei der Palpation der Kiefergelenke von lateral ausgeübten Drucks wird eine Erhöhung der in den RDC/TMD empfohlenen Werte von ca. $5 \mathrm{~N} / \mathrm{cm}^{2}$ auf ca. $15 \mathrm{~N} / \mathrm{cm}^{2}$ empfohlen; dadurch sollen eine gleichermaßen hohe Sensitivität und Spezifität für die Diagnose „Arthralgie“ erzielt werden [75]. Eine vorherige Kalibrierung des Untersuchers mit Hilfe einer Waage ist notwendig.

Okklusale Befundung. Im Rahmen der Okklusionsdiagnostik werden folgende Parameter erfasst:

- Zahnstatus,

- Zahnkontakte in habitueller/maximaler Interkuspidation (statische Okklusion),

- Attritionen und keilförmige Defekte.

Beim Zahnstatus sind die vorhandenen bzw. fehlenden Zähne zu notieren, weil es Hinweise dafür gibt, dass ein vollständiger Verlust der Seitenzahnabstützung ein Risikofaktor für schmerzhafte Kiefergelenkbeschwerden sein kann [5, 6, 70, 73].

Durch die Bestimmung des Zahnstatus und der vorhandenen Zahnkontakte in statischer Okklusion werden die ausreichende Abstützung der Kiefer im Seitenzahnbereich überprüft und mögliche Bereiche einer Nonokklusion erfasst.

Zähneknirschen und/oder Kieferpressen (Bruxismus) gelten als Risikofaktoren für Kiefermuskelschmerzen [31, 95]. Zähneknirschen führt darüber hinaus zu Attrition (Zahnhartsubstanzverlust aufgrund von Zahn-zu-Zahn-Kontakten). Häufige Folge dieser Parafunktionen ist ein spaltfreies Aufeinanderliegen der Schneide- und Eckzähne, wenn der Unterkiefer unter Zahnkontakt nach vorne und zur Seite geschoben wird, bis sich die Schneidekanten berühren. Ein solcher Befund gilt als klinisches Zeichen für Knirschen. Ein weiterer möglicher Hinweis für Bruxismus sind keilförmige Defekte im Zahnhalsbereich (sog. Abfraktionen oder nichtkariöse zervikale Läsionen [68]).

\section{Panoramaschichtaufnahme}

Die Anfertigung einer Panoramaschichtaufnahme (Orthopantomogramm) stellt bei Patienten mit vermuteten Myoarthro-

Schmerz 2006 $20: 481-489$ DOI 10.1007/s00482-006-0472-9

(C) electronic version (html) Springer Medizin Verlag 2006

(๑) print \& online pdf Deutsche Gesellschaft zum Studium des Schmerzes 2006

Published by Springer Medizin Verlag - all rights reserved

J. C. Türp · A. Hugger · P. Nilges · S. Hugger · J. Siegert - E. Busche · S. Effenberger H. J. Schindler

\section{Aktualisierung der Empfehlungen zur standardisierten Diagnostik und Klassifikation von Kaumuskel- und Kiefergelenkschmerzen}

Zusammenfassung

Hintergrund. Im Jahre 2000 veröffentlichte der Interdisziplinäre Arbeitskreis für Mundund Gesichtsschmerzen in der Deutschen Gesellschaft zum Studium des Schmerzes Empfehlungen zur Diagnostik und Klassifikation von Patienten mit Schmerzen im Bereich der Kaumuskulatur und/oder Kiefergelenke. Ziele der vorliegenden Publikation sind eine Bestandsaufnahme und Aktualisierung der damals gemachten Vorschläge.

Ergebnisse. Sichtung und Bewertung der nach Veröffentlichung der Empfehlungen erschienenen Fachliteratur (bis Dezember 2005) zeigen, dass sich das zweiachsige Stufenkonzept zur Erfassung somatischer und psychosozialer Parameter orofazialer Schmer- zen bewährt hat. Einzelne Aspekte der Empfehlungen wurden in Form wissenschaftlicher Belege weiter konkretisiert. Schlussfolgerungen. Die vorgeschlagenen Empfehlungen spiegeln die aktuellen Entwicklungen in der Schmerzmedizin wider. Insbesondere in der Zahnärzteschaft sollten sie daher eine noch breitere Verankerung finden als bisher.

\section{Schlüsselwörter}

Kraniomandibuläre Dysfunktionen · Myoarthropathien · Gesichtsschmerzen · Schmerzdiagnostik $\cdot$ Klassifikationssystem

\section{Recommendations for the standardized evaluation and classification of painful temporomandibular disorders: an update}

\section{Abstract}

Background. In 2000, the interdisciplinary Working Group of Orofacial Pain within the German Chapter of the IASP (German Association for the Study of Pain) published recommendations for the standardized evaluation and diagnostic classification of patients with painful masticatory muscles and/or temporomandibular joints. The purpose of the present study was to critically review and update these recommendations.

Results. Appraisal of the relevant literature published after the release of the recommendations (up to December 2005) shows that the two-axis approach for the assessment of the somatic and psychosocial parameters of the orofacial pain experience has found wide support. Single aspects of the recommendations have been substantiated by additional scientific evidence.

Conclusions. The recommendations reflect the current state of pain medicine. Therefore, they should be considered among practitioners who diagnose and manage patients suffering from temporomandibular disorders.

\section{Keywords}

Temporomandibular disorders · Craniomandibular disorders - Orofacial pain · Pain evaluation. Classification system 
pathien eine sehr sinnvolle und empfehlenswerte differenzialdiagnostische Maßnahme dar $[53,56]$. Aufgrund der großen Bedeutung der Panoramaschichtaufnahme für die Differenzialdiagnostik wird eine solche Übersichtsaufnahme bereits in der Initialphase des diagnostischen Vorgehens, d. h. im Rahmen der Mindestdiagnostik, obligat angefertigt.

Für die eigentliche Diagnose spielen die Röntgenbefunde dagegen nur eine geringe Rolle [15]. Speziell im Kiefergelenkbereich sollte man sich vor einer Überinterpretation der morphologischen Befunde hüten: Knöcherne Veränderungen, wie Abflachung der knöchernen Gelenkflächen oder Osteophytenbildung, sind bei Patienten mit myoarthropathischen Schmerzen genau so häufig festzustellen wie bei Personen ohne solche Beschwerden [7].

Vor allem im Hinblick auf den vor kurzem geäußerten Hinweis auf die hohe Patientenexposition bei der Strahlendiagnostik in Deutschland und anderen Ländern $[2,29,69]$ erscheint das günstige Nutzen-Risiko-Verhältnis für Panoramaschichtaufnahmen erwähnenswert: Bei vergleichsweise geringer Strahlenbelastung des Patienten (effektive Dosis 0,004-0,03 $\mathrm{mSv}[16]$ ) ist der Informationsgehalt hoch.

\section{Psychologische Filterfragebögen}

Im Rahmen der Mindestdiagnostik erfolgt eine Abschätzung, ob ein sog. dysfunktionaler Schmerz vorliegt, mit Hilfe der Graduierung chronischer Schmerzen (GCS). Bei der GCS handelt es sich um die deutsche Version des international weit gebräuchlichen Graded Chronic Pain Status [97]. Die GCS ist Teil des erwähnten 14-seitigen Schmerzfragebogens. Sie kann bei Bedarf auch gesondert eingesetzt werden, z. B. zur diagnostischen Abschätzung des therapeutischen Erfolgs [12, 13]; ein entsprechender GCS-Bogen mit ausführlicher Anleitung wurde in einer weit verbreiteten deutschsprachigen zahnärztlichen Fachzeitschrift vorgestellt [84]. Im neuen DGSS-Schmerzfragebogen ist die GCS ebenfalls enthalten [58]. Im Rahmen einer Untersuchung von Patienten einer speziellen Myoarthropathie-Ambulanz konnte mit Hilfe der GCS gezeigt werden, dass sich ca. $25 \%$ der $\mathrm{Pa}$ tienten mit dysfunktional einzustufenden chronischen Schmerzen vorstellten und damit die Notwendigkeit einer nicht nur somatisch orientierten Befunderhebung und Behandlung gegeben ist [62].

Aufgrund unserer sehr engen Anlehnung an die RDC/TMD haben wir die GCS der Mainzer Stadieneinteilung des Schmerzes [21, 22], das im deutschen Sprachraum ein anerkanntes Verfahren darstellt, vorgezogen.

\section{Standarddiagnostik}

Im Rahmen der Standarddiagnostik kommen die Allgemeine Depressionsskala (ADS [28]) und die Beschwerden-Liste (B-L [98]) zur Anwendung. Die Gütekriterien Objektivität, Reliabilität und Validität dieser beiden Instrumente werden als hoch eingeschätzt [3]:

1. Bei beiden Tests erfolgen Bearbeitung und Auswertung unabhängig vom Untersucher, somit ist die Objektivität vorhanden.

2. ADS und B-L weisen eine hohe Reliabilität auf.

3. Beide Tests besitzen eine ausreichende Validität.

Eine Studie an 95 Patienten mit persistierenden muskuloskelettalen Gesichtsschmerzen ergab, dass der möglichst frühzeitige Einsatz von ADS und B-L zusätzlich zur GCS sinnvoll erscheint [1]. Daher sollte man die Indikation für eine Standarddiagnostik möglichst großzügig stellen, zumal das Ausfüllen und Auswerten beider Bögen nur wenige Minuten in Anspruch nimmt.

\section{Erweiterte Diagnostik}

Für weitere wichtige Aspekte von Schmerz wie Behinderung sowie affektives und sensorisches Erleben sind geeignete und zuverlässige Fragebögen vorhanden.

Zur Einschätzung des Grades der subjektiven Beeinträchtigung lässt sich der mit 7 Items kurze Pain Disability Index (PDI $[9,81]$ ) als Screening-Verfahren verwenden. Im Vergleich zu anderen Fragebögen ist der PDI weniger syndromabhängig, d. h. er ist bei Patienten mit Gesichtsschmerzen ebenso verwendbar wie bei anderen Schmerzlokalisationen [40].

Die sensorischen und affektiven Dimensionen des Schmerzes können zuverlässig mit der Schmerzempfindungsska- la (SES [20]) erfasst werden. Die affektive Dimension beschreibt den „Leidensaspekt“; enge Beziehungen zu Angst, Depression und Hilflosigkeit sind feststellbar. Die SES wird in der überarbeiteten Fassung des neuen DGSS-Schmerzfragebogens unverändert enthalten sein.

\section{Weitere bildgebende Verfahren}

Die Notwendigkeit der Anwendung bildgebender Verfahren über die Panoramaschichtaufnahme hinaus, z. B. transkraniale Aufnahmen, konventionelle Tomographie, Computer- oder Magnetresonanztomographie der Kiefergelenke [35], ist eingeschränkt. Ihr Einsatz sollte immer mit individuellen Informationen aus der Anamnese und/oder der klinischen Untersuchung verbunden sein und auf begründete Einzelfälle (z. B. Tumorverdacht) beschränkt bleiben [53]. Die Beziehung zwischen bildgebenden Befunden und Schmerzangaben aus dem Bereich der Kiefergelenke ist trotz erheblicher Verbesserungen bei der Visualisierung arthrogener Strukturen mit Hilfe computerunterstützter Techniken sehr komplex [33]. Die isolierte Beurteilung von Gelenkstrukturen mit ihrer Vielfalt der Form- und Positionsausprägung liefert keinen ausreichend scharf abgrenzbaren Vorhersagewert, um gesund von krank sowie schmerzhaft von nichtschmerzhaft zu trennen. Bei Patienten mit gelenkbezogenem Schmerz bzw. Dysfunktion oder Verdacht auf Diskusverlagerung sollte erst dann an die Anfertigung von Magnetresonanztomogrammen gedacht werden, wenn sich die Symptome refraktär gegenüber konservativen Behandlungsmaßnahmen verhalten [4]. Wenig zweckmäßig erscheint ebenfalls der Einsatz bildgebender Verfahren im Stadium der Initialtherapie bei muskulär bezogenen Schmerzen; erst zum Zeitpunkt persistierender Schmerzen, die konservativen Behandlungsmaßnahmen nicht zugänglich sind, öffnet sich das Einsatzgebiet für die Hartbzw. Weichgewebebildgebung.

\section{Klinische und instrumentelle Okklusionsanalyse}

Die Bedeutung der Okklusion für Prädisponierung, Auslösung bzw. Aufrechterhaltung von Kaumuskel- und/oder Kiefergelenkschmerzen ist bis heute Gegenstand der wissenschaftlichen Diskussion. Wäh- 
Hier steht eine Anzeige.

第 Springer 


\section{Übersichten}

\begin{tabular}{|c|c|}
\hline Maßnahme & Methode/Instrument \\
\hline \multicolumn{2}{|l|}{ Mindestdiagnostik } \\
\hline \multirow[t]{2}{*}{ Schmerzbezogene Anamnese } & Persönliches Gespräch \\
\hline & $\begin{array}{l}\text { Fragebögen mit Ganzkörperzeichnung, z. B. } \\
\text { „Schmerzfragebogen für Patienten mit } \\
\text { chronischen orofazialen Schmerzen“ [87] }\end{array}$ \\
\hline Graduierung chronischer Schmerzen & $\begin{array}{l}\text { Fragebogen „Graduierung chronischer } \\
\text { Schmerzen“ [84] }\end{array}$ \\
\hline \multicolumn{2}{|l|}{ Klinische Befundung } \\
\hline Kieferbeweglichkeit & Lineal \\
\hline Palpationsempfindlichkeit & Palpation mit Finger \\
\hline Okklusion & $\begin{array}{l}\text { Visuelle Inspektion, Shimstock-Folie im } \\
\text { Folienhalter }\end{array}$ \\
\hline Röntgenübersichtsaufnahme & Panoramaschichtaufnahme \\
\hline \multicolumn{2}{|l|}{ Standarddiagnostik } \\
\hline Screening: Depressive Verstimmung & Allgemeine Depressionsskala [28] \\
\hline Screening: Unspezifische Beschwerden & Beschwerden-Liste [99] \\
\hline
\end{tabular}

rend in früheren Jahrzehnten die Okklusion als der zentrale Faktor in diesem Geschehen angesehen wurde, musste ihre Bedeutung auf der Grundlage einer Reihe von Untersuchungen deutlich relativiert werden, ohne ihr jedoch eine Einflussnahme gänzlich abzusprechen $[23,24,25,36,46$, 66]. Im Rahmen des diagnostischen Prozesses bei Patienten mit Kaumuskel- und Kiefergelenkschmerzen muss beachtet werden, dass bei der Beurteilung der individuellen okklusalen Situation zum einen allzu abstrakte ideale Vorstellungen von der "Norm" keine ausschlaggebende Bedeutung erlangen [102]. Zum anderen liegen zahlreiche Nachweise dafür vor, dass es in der Phase des Schmerzes durch sensomotorische Reaktionen (Schmerz-Adaptationsmodell [50]) zu Veränderungen der Unterkieferlage und Bewegungsausführung kommt, was sich auf den Zusammenbiss (Okklusion) der Zähne auswirken kann [51]. Damit wird es in der Phase des eigentlichen Schmerzzustands nahezu unmöglich, zwischen Ursache und Wirkung im Hinblick auf die gegebene okklusale Situation zu unterscheiden [9o].

Eine suffiziente klinische wie auch instrumentelle Okklusionsanalyse erscheint daher, wenn überhaupt, erst nach erfolgter Schmerztherapie sinnvoll. Durch die okklusale Analyse soll versucht werden, mögliche Störfaktoren (etwa in Form von Vorkontakten oder Gleithindernissen) kenntlich zu machen und Hinweise auf okklusale Parafunktionen zu geben. Ziel ist es, durch adäquate Einstellung und Stabilisierung der Unterkieferausgangsposition sowie durch Gewährleistung eines angepassten Bewegungsfreiraums zu einer biomechanischen Optimierung sowie zu einer schmerz- und dysfunktionsbezogenen Risikominimierung beizutragen $[38,61]$.

Die klinische Okklusionsanalyse erlaubt die Beurteilung der patientenbezogenen okklusalen Situation aus eingeschränkter Perspektive [64]. In Form der instrumentellen Okklusionsanalyse werden diese visuellen Einschränkungen zwar überwunden, jedoch ergeben sich durch die erforderliche Verwendung bestimmter Werkstoffe und Geräte Folgerungen, die eine unkritische Umsetzung der instrumentell gewonnenen Erkenntnisse auf die In-vivo-Situation durchaus problematisch erscheinen lassen [43, 77].

\section{Manuelle Funktionsanalyse}

Neben wichtigen Untersuchungselementen im Rahmen der klinischen Funktionsanalyse - v. a. Messung der Unterkiefermobilität, Palpation der Kiefermuskulatur, Palpation bzw. Auskultation der Kiefergelenke sind weitere klinische Untersuchungsverfahren bzw. Tests im Rahmen der manuellen Funktionsanalyse bekannt. Hierzu zählen so genannte orthopädische Tests wie - Endgefühl („,end feel“, Vergleich der aktiven und passiven Unterkieferbeweglichkeit),
- Gelenkspiel („joint play“, Ausführung von Traktions- bzw. Translationsbewegungen im Kiefergelenkbereich),

- Kompressionstest (Ausübung von kranial gerichtetem Druck im Kieferwinkelbereich) und

- statische bzw. dynamische Widerstandstests (Ausführung von Unterkieferbewegungen gegen Widerstand).

Die wesentliche Intention dieser speziellen Tests ist es, strukturbezogen zwischen myogener, arthrogener oder myoarthrogener Genese der Myoarthropathie zu differenzieren [30, 49]. Hinsichtlich der Reliabilität, Validität und diagnostischen Bedeutung der Tests liegen bis heute kaum aussagekräftige Studien vor. Bisherige Studienergebnisse lassen erkennen, dass die genannten Tests offenbar eine höhere Spezifität als Sensitivität aufweisen, aber zur Sicherstellung einer ausreichenden Zuverlässigkeit der Tests eine intensive Schulung mit Training in regelmäßigen Zeitabständen besonders erforderlich erscheint $[8,10]$.

\section{Instrumentelle Funktionsanalyse}

Unter dem Begriff „Instrumentelle Funktionsanalyse“ wird die „dynamische Aufzeichnung der Unterkieferbewegungen und deren diagnostische Interpretation" verstanden. Für die Aufzeichnung von Bewegungsspuren des Unterkiefers bzw. der Unterkieferkondylen (Achsio-/Axiographie) werden neben graphisch-mechanischen Registrierverfahren zunehmend computerunterstützte elektronische Messsysteme verwendet $[32,41,42]$.

Der Stellenwert der instrumentellen Funktionsdiagnostik im Rahmen der Schmerzdiagnostik bleibt umstritten [44, 90]. Während ein Teil der Zahnärzteschaft der Meinung ist, dass im Rahmen der Diagnostik myoarthropathischer Schmerzen auf die Verwendung solcher Registriersysteme verzichtet werden kann (Zusammenfassung der Argumente bei [88]), hält ein anderer Teil, darunter deutsche zahnärztliche Fachgesellschaften, deren Einsatz für sinnvoll [14].

Es muss betont werden, dass die instrumentelle Funktionsdiagnostik nicht der Erfassung orofazialer Schmerzen dient. Stattdessen lassen sich mit den eingesetzten, zumeist elektronischen Geräten - ausreichende Reliabilität und Vali- 
dität vorausgesetzt [34] - sekundäre Auswirkungen der Schmerzen auf die Unterkieferfunktion (z. B. Einschränkungen der Unterkieferbeweglichkeit, Dyskoordination, Vermeidungsstrategien im Bewegungsablauf, Schonhaltung) im Sinne der Objektivierung des individuellen Funktionszustands bestimmen und eine objektivierende Verlaufskontrolle (bei funktionstherapeutischen Maßnahmen, zur Qualitätskontrolle) durchführen.

In Kombination mit geeigneten bildgebenden Verfahren kann für den Einzelfall ferner vor dem Hintergrund eines Schmerzgeschehens der Grad des Zusammenhangs zwischen struktur- und funktionsbezogenen Befunden ermittelt werden. In dieser Hinsicht können entsprechende Registriersysteme sinnvoll eingesetzt werden. In welchem Ausmaß die aus der instrumentellen Funktionsdiagnostik gewonnenen Ergebnisse und deren diagnostische Folgerungen die Diagnosestellung, Behandlungsentscheidung, Prognoseermittlung und Sicherung des Therapieverlaufs gegenüber den Befunden aus rein klinischer Untersuchung in grundsätzlicher Weise beeinflussen, bedarf weiterer Klärung.

\section{Fazit für die Praxis}

Die vorgeschlagenen Empfehlungen spiegeln die aktuellen Entwicklungen in der wissenschaftlichen Fachliteratur wider. Im anbrechenden Zeitalter der evidenzbasierten (Zahn-)Medizin sollten sie daher insbesondere in der Zahnärzteschaft eine noch breitere Verankerung finden, als dies bislang der Fall gewesen ist. Empfehlungen zur Therapie von Kaumuskel- und Kiefergelenkschmerzen werden vom Interdisziplinären Arbeitskreis für Mund- und Gesichtsschmerzen derzeit erarbeitet und in Kürze in dieser Zeitschrift vorgestellt.

\section{Korrespondierender Autor Prof. Dr. J.C. Türp}

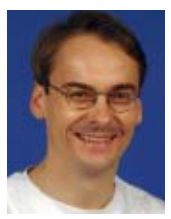

Klinik für Rekonstruktive Zahnmedizin und Myoarthropathien, Universitätskliniken für Zahnmedizin Hebelstrasse 3, 4056 Basel jens.tuerp@unibas.ch
Interessenkonflikt. Es besteht kein Interessenkonflikt. Der korrespondierende Autor versichert, dass keine Verbindungen mit einer Firma, deren Produkt in dem Artikel genannt ist, oder einer Firma, die ein Konkurrenzprodukt vertreibt, bestehen. Die Präsentation des Themas ist unabhängig und die Darstellung der Inhalte produktneutral.

\section{Literatur}

1. Becker INS, Türp JC, Gerds T (1999) Psychosoziale Filterdiagnostik bei Patienten mit chronischen $\mathrm{Ge}$ sichtsschmerzen. Schmerz 15 [Suppl 1]: S82-83 (P11.82)

10. Dittmann R, Houshmand M, Schwahn C et al. (2003) Interexaminer reliability of different clinical tests for the assessment of arthrogenous TMD signs. J Dent Res 82: B-232, abstr \#1766

11. Dworkin SF, LeResche L (1992) Research diagnostic criteria for temporomandibular disorders: review, criteria, examinations and specifications, critique. J Craniomandib Disord Facial Oral Pain 6: 301-355

12. Dworkin SF, Huggins KH, Wilson L et al. (2002) A randomized clinical trial using research diagnostic criteria for temporomandibular disorders-axis II to target clinic cases for a tailored self-care TMD treatment program. J Orofac Pain 16: 48-63

13. Dworkin SF, Sherman J, Mancl L et al. (2002) Reliability, validity, and clinical utility of the research diagnostic criteria for temporomandibular disorders axis II scales: depression, non-specific physical symptoms, and graded chronic pain. J Orofac Pain 16: 207-220

14. Engelhardt JP, Hugger $A$, Jakstat $H$, Kordaß B (2002) Instrumentelle Funktionsanalyse. Gemeinsame Stellungnahme der Deutschen Gesellschaft für Zahn-, Mund- und Kieferheilkunde, der Deutschen Gesellschaft für Zahnärztliche Prothetik und Werkstoffkunde und der Arbeitsgemeinschaft für Funktionslehre in der DGZMK. Dtsch ZahnärztI Z 57: 659

18. Freesmeyer WB, Fussnegger MR, Ahlers MO (2003) Aspekte in der Diagnostik und Therapie von CMDPatienten. Dtsch Zahnärztekal 2004: 221-245

24. Gesch D, Bernhardt O, Kocher T et al. (2004) Association of malocclusion and functional occlusion with signs of temporomandibular disorders in adults: results of the population-based study of health in Pomerania. Angle Orthod 74: 512-520

25. Gesch D, Bernhardt O, Mack F et al. (2005) Association of malocclusion and functional occlusion with subjective symptoms of TMD in adults: results of the Study of Health in Pomerania (SHIP). Angle Orthod 75: 183-190

31. Huang GJ, LeResche L, Critchlow CW et al. (2002) Risk factors for diagnostic subgroups of painful temporomandibular disorders (TMD). J Dent Res 81: 284-288

33. Hugger A (2002) Bildgebende Diagnostik bei Schmerzsymptomatik im Kiefergelenkbereich. Schmerz 16: 355-364

46. Le Bell Y, Jämsä T, Korri S et al. (2002) Effect of artificial occlusal interferences depends on previous experience of temporomandibular disorders. Acta Odontol Scand 60: 219-222

48. Leder S (2005) Funktionsstörungen erkennen und behandeln. Spitta, Balingen

62. Paak S, Hugger A, Bollmann F, Stüttgen U (2001) Schmerzbezogene psychosoziale Aspekte bei Patienten der Myoarthropathie-Sprechstunde. Dtsch Zahnärztl Z 56: 317-312
65. Palla S (2003) Myoarthropathischer Schmerz des Kausystems. In: Mense S, Pongratz D (Hrsg) Chronischer Muskelschmerz. Steinkopff, Darmstadt, S 145-166

66. Pullinger AG, Seligman DA (2000) Quantification and validation of predictive values of occlusal variables in temporomandibular disorders using a multifactorial analysis. J Prosthet Dent 83: 66-75

67. Rantala MA, Ahlberg J, Suvinen Tl et al. (2003) Symptoms, signs, and clinical diagnoses according to the research diagnostic criteria for temporomandibular disorders among Finnish multiprofessional media personnel. J Orofac Pain 17: 311-316

70. Sarita PT, Kreulen CM, Witter D, Creugers NH (2003) Signs and symptoms associated with TMD in adults with shortened dental arches. Int J Prosthodont 16: 265-270

71. Schmitter M, Leckel M, Rammelsberg P (2003) 2Achsen-System der RDC/TMD zur standardisierten Diagnostik von Funktionsstörungen (Teil 1): Die klinische Untersuchung. Zahnärztebl Bad Württ 31(9): 16-19

73. Seedorf H, Seetzen F, Scholz A et al. (2004) Impact of posterior occlusal support on the condylar position. J Oral Rehabil 31: 759-763

75. Shaefer JR, Jackson DL, Schiffman EL, Anderson QN (2001) Pressure-pain thresholds and MRI effusions in TMJ arthralgia. J Dent Res 80: 1935-1939

83. Türp JC, John M, Nilges P et al. (2000) Schmerzen im Bereich der Kaumuskulatur und Kiefergelenke. Empfehlungen zur standardisierten Diagnostik und Klassifikation von Patienten. Schmerz 14: 416-428

84. Türp JC, Nilges P (2000) Diagnostik von Patienten mit chronischen orofazialen Schmerzen. Die deutsche Version des "Graded Chronic Pain Status". Quintessenz 51: 721-727

87. Türp JC, Marinello CP (2002) Schmerzfragebogen für Patienten mit chronischen orofazialen Schmerzen. Quintessenz 53: 1333-1340, http://www. quintessenz.de

88. Türp JC (2003) Ist die instrumentelle Funktionsanalyse eine empfehlenswerte Maßnahme zur Diagnostik kraniomandibulärer Funktionsstörungen? Dtsch Zahnärztl Z 58: 477-481

94. Türp JC, Schindler HJ, Bartzela T (2005) Schmerzhafte Myoarthropathien des Kausystems - evidenzbasierte Diagnostik. Kieferorthop 19: 173181

95. Velly AM, Gornitsky M, Philippe P (2003) Contributing factors to chronic myofascial pain: a case-control study. Pain 104: 491-499

96. Vollaro S, Michelotti A, Cimino R et al. (2001) Epidemiologic study of patients with temporomandibular disorders. Report of data and clinical findings. Minerva Stomatol 50: 9-14

99. Wirz S, Wartenberg HC, Wittmann M et al. (2003) Versorgung von Patienten mit chronischem orofazialem Schmerz. Ergebnisse einer Befragung in ambulanten zahnärztlichen und Mund-Kiefer-Gesichts-Chirurgie-Einrichtungen. Schmerz 17: 325 331

100. Yap AU, Dworkin SF, Chua EK et al. (2003) Prevalence of temporomandibular disorder subtypes, psychologic distress, and psychosocial dysfunction in Asian patients. J Orofac Pain 17: 21-28

Das komplette Literaturverzeichnis ..

... finden Sie in der elektronischen Version dieses Beitrags unter www.DerSchmerz.de 